\title{
Solitary intraventricular hemorrhage without subarachnoid hemorrhage due to aneurysmal rupture: a case report
}

\author{
Seung Soo $\mathrm{Kim}^{1 \dagger}$, Kyeong-O Go ${ }^{\dagger}$, Hyun Park ${ }^{1 *}$, Kwangho Lee $^{3}$, Gyeong Hwa Ryu ${ }^{4}$, Hye Jin Baek ${ }^{4}$, \\ Seunguk Jung ${ }^{5}$, Chang Hyo Yoon ${ }^{5}$, Young-Seop Park', Soo Hyun Hwang' \\ 'Department of Neurosurgery, Gyeongsang National University School of Medicine and Gyeongsang National \\ University Changwon Hospital, Changwon, South Korea, ${ }^{2}$ Department of Neurosurgery, Seoul National University \\ Bundang Hospital, Seongnam-si, South Korea, ${ }^{3}$ Department of Neurosurgery Gyeongsang National University School \\ of Medicine and Gyeongsang National University Hospital, Jinju, South Korea, ${ }^{4}$ Department of Radiology, \\ Gyeongsang National University School of Medicine and Gyeongsang National University Changwon Hospital, \\ Changwon, South Korea, ${ }^{5}$ Department of Neurology, Gyeongsang National University School of Medicine and \\ Gyeongsang National University Changwon Hospital, Changwon, South Korea \\ t. Contributed equally authorized
}

The presence of primary intraventricular hemorrhage (IVH) without vascular lesion is very rare. We experienced solitary IVH without subarachnoid hemorrhage due to aneurysmal rupture in a 58-year-old man treated with coil embolization, which contributed to his good prognosis. After 33 days of hospitalization, he had mild right hemiplegic symptoms remaining, and he was transferred to a rehabilitation institute for further treatment. In cases of primary IVH, computed tomography angiography seems worthwhile for making a differential diagnosis, although the possibility of IVH due to cerebral aneurysmal rupture is very low. Endovascular intervention is a good option for diagnosis and treatment.

Keywords Cerebral angiography; Intraventricular hemorrhage; Intracranial aneurysm; Endovascular procedures; Subarachnoid hemorrhage
J Cerebrovasc Endovasc Neurosurg. 2019 March;21(1):18-23

Received : 7 January 2019

Revised : 16 February 2019

Accepted : 23 March 2019

\section{Correspondence to Hyun Park}

Department of Neurosurgery, Gyeongsang National University Changwon Hospital, Gyeongsang National University College of Medicine, 11, Samjeongja-ro, Seongsan-gu, Changwon-si, Gyeongsangnam-do, South Korea, 51472

\author{
Tel : $+82-55-214-2440$ \\ Fax : +82-55-214-1031 \\ E-mail : 1coo3004@naver.com \\ ORCID : http://orcid.org/0000-0002-2422-4389
}

This is an Open Access article distributed under the terms of the Creative Commons Attribution NonCommercial License /http://creativecommons.org/licenses/by-nc/3.0) which permits unrestricted noncommercial use, distribution, and reproduction in any medium, provided the original work is properly cited.

\section{INTRODUCTION}

Intraventricular hemorrhages (IVHs) are usually secondary to intra-parenchymal hemorrhages (IPHs) adjacent to ventricles or subarachnoid hemorrhages (SAHs), and head trauma is a frequent cause of IVH. However, primary IVH without any evidence of SAH or IPH on a non-contrast-enhanced computed tomography (CT) scan is very rare, accounting for $3 \%$ of all intracranial hemorrhages. ${ }^{4,21)}$ In many previous cases, vascular abnormalities such as arteriovenous malformations (AVMs) and cerebral aneurysmal ruptures, could have been the cause of bleeding. ${ }^{4)}$ To the best our knowledge, there are very rare cases of solitary IVH without SAH result from aneurysmal ruptures. $1,6,8,9,11,12,13,19,26)$ We describe our experience with diagnosis and treating an extremely rare case of solitary IVH without SAH due to aneurysmal rupture. 


\section{Description of Case}

A 58-year-old man was transferred to the emergency room because of headache, dizziness, and altered mentality; his initial Glasgow coma scale score was 13 . He had no history of hypertension. He provided inappropriate responses to questions and showed memory disturbance. He also had right-sided weakness of motor grade IV.

The initial brain CT scan revealed solitary IVH and mild enlargement of the left lateral ventricle, and the hemorrhage expanded through the foramen Monro to the contralateral anterior horn of the lateral and third ventricles (Fig. 1A, B). For a differential diagnosis, CT angiography (CTA) was performed and revealed a ruptured aneurysm in the left proximal anterior choroidal artery (Fig. 2A, B). Immediate conventional angiography was performed and showed a ruptured 7-mm left anterior choroidal artery aneurysm with bleb (Fig. 3A-C). The aneurysm was sequentially embolized with an endovascular coil. Then, the drainage of cerebrospinal fluid (CSF) was done through a lumbar drainage catheter rather than through external ventricular drainage (EVD) for 7 days to facilitate washout of IVH (Fig. 4A, B).

Transcranial Doppler ultrasonography was performed daily for 10 days and revealed no evidence of vasospasms. After 33 days of hospitalization, the patient had mild right hemiplegic symptoms remaining, and he was transferred to a rehabilitation institute for further treatment.

\section{Discussion}

Solitary IVHs, non-traumatic spontaneous IVHs without SAH or IPH, are very rare. ${ }^{2,3,5,7)}$ Reportedly, solitary IVH accounts for $1 \%$ to $3.1 \%$ and up to 8.8 $\%$ of all non-traumatic intracranial hemorrhages. ${ }^{3,7,24)}$ IVHs manifesting secondary to a cerebral vascular lesion or expanding from the parenchymal hemorrhage near ventricles occur more often. ${ }^{24)}$ However, the actual incidence of primary IVH is unclear because primary IVH may be one of the causes of sudden death, occurring before the patient reaches the hospital or before brain imaging evaluation can be performed. ${ }^{10)}$ In most cases, cerebral aneurysmal rupture manifests with SAHs, and even acute subdural hemorrhages, IPHs and IVHs. ${ }^{14,15,18)}$ About $2 \%$ of radiologically diagnosed SAHs were associated with IVH. ${ }^{1)}$ Additionally, isolated IVH caused by cerebral aneurysmal rupture without SAH is extremely rare $(0.12 \%)$ and associated with a relatively poor outcome. ${ }^{1)}$

Previous studies revealed that hypertension is the most common contributing etiology of primary IVH, and in cases of no vascular lesion, diabetes, hyperlipidemia and coagulopathies were other contributing factors. ${ }^{2,520,23)}$ Vascular abnormalities such as AVMs and aneurysms, were the second most common cause, and the other causes remain unknown. ${ }^{23)}$ In some previous case series, vascular malformation and aneurysms were considered the most common causes, accounting for $25-57 \%$ of cases. $2,3,7,16,20)$

Further evaluation is needed to reveal other vascular abnormalities. Many authors performed conventional magnetic resonance angiography, CTA, or digital subtraction angiography (DSA). ${ }^{1,2,3,4,21,24)}$ However, clinically, even if IVH is confirmed on the initial CT scan, diagnostic angiography would not be performed in patients whose mortality is expected to be very high because of their general condition or in those with a poor level of consciousness. However, if possible, diagnostic angiography should be performed to confirm vascular lesions that cause bleeding and to prevent the risk of recurrence. In our institute, CTA is performed in case of isolated IVH as a routine evaluation

CTA is non-invasive and requires minimal additional time and cost, and it is associated with a lower complication rate than DSA. ${ }^{25)}$ The overall sensitivity, specificity, positive predictive value, negative predictive value and accuracy of CTA were $89 \%, 92 \%$, $91 \%, 91 \%$ and $91 \%$, respectively. ${ }^{25}$ ) Nevertheless, two of four dural arteriovenous fistulas were missed by CTA examination. ${ }^{25}$ In case of spontaneous intracerebral hemorrhage other than SAH, it is necessary 

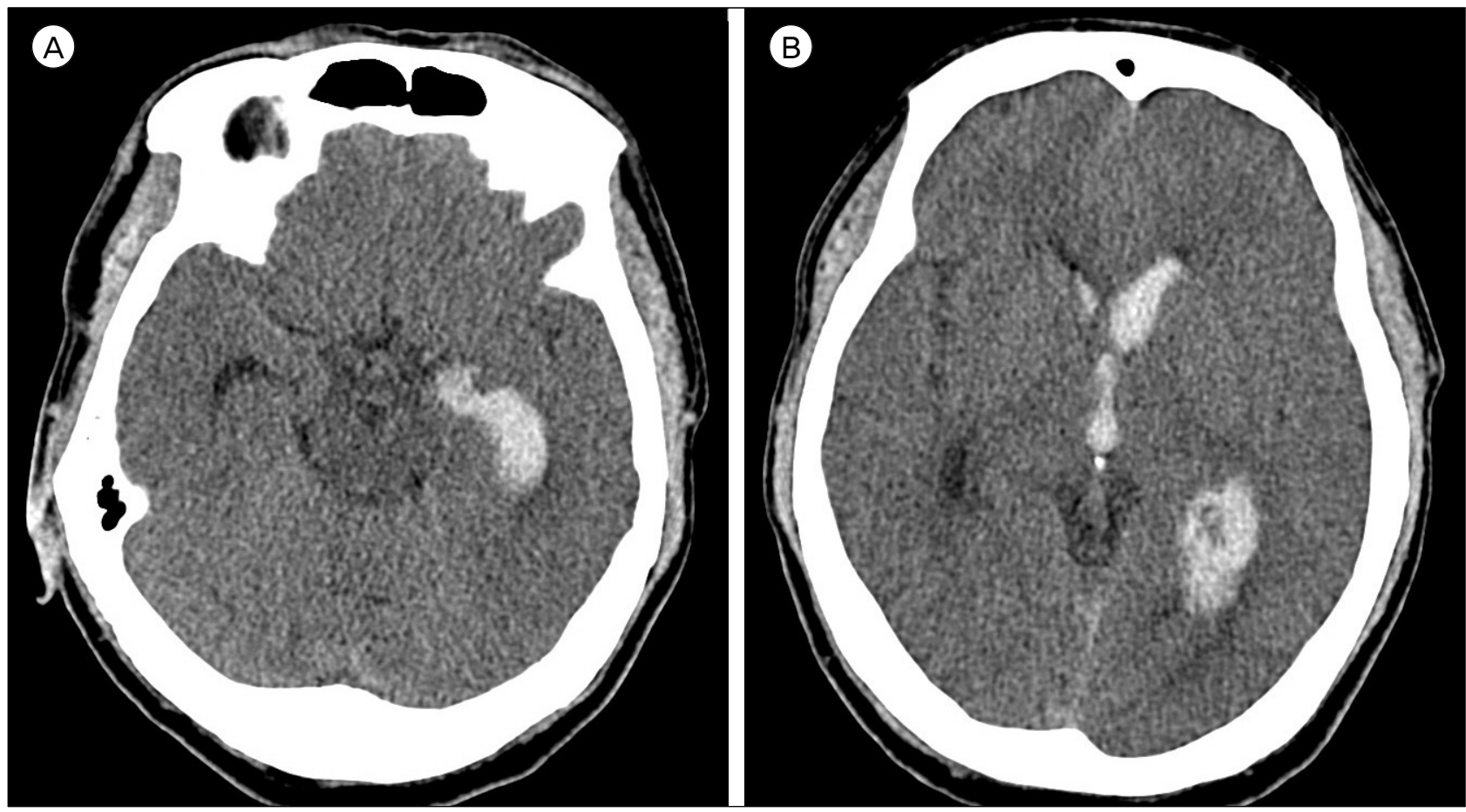

Fig. 1. The initially checked non-contrast-enhance computed tomography scans. A: The hemorrhage is expanded through the foramen Monro to the contralateral anterior horn of the lateral and third ventricles. B: No definite subarachnoid hemorrhage is observed on the initial computed tomography scan.
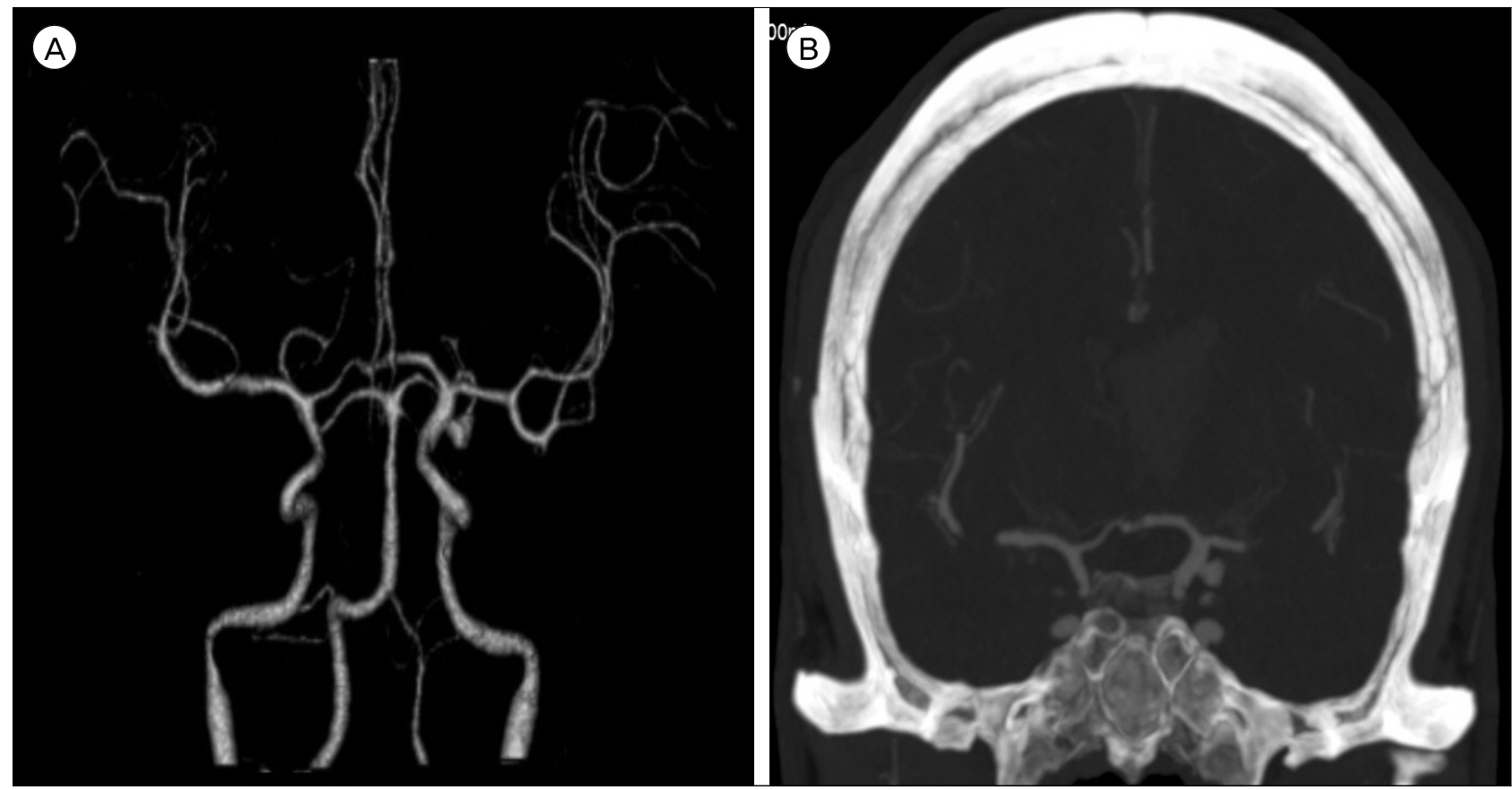

Fig. 2. Computed tomography angiography is performed for the differential diagnosis. A: Three-dimensional reconstruction image shows the aneurysm in left distal internal carotid artery. B: Computed tomography angiogram of the coronal section.

to perform further evaluation for the differential diagnosis of vascular lesion and a sequential treatment 


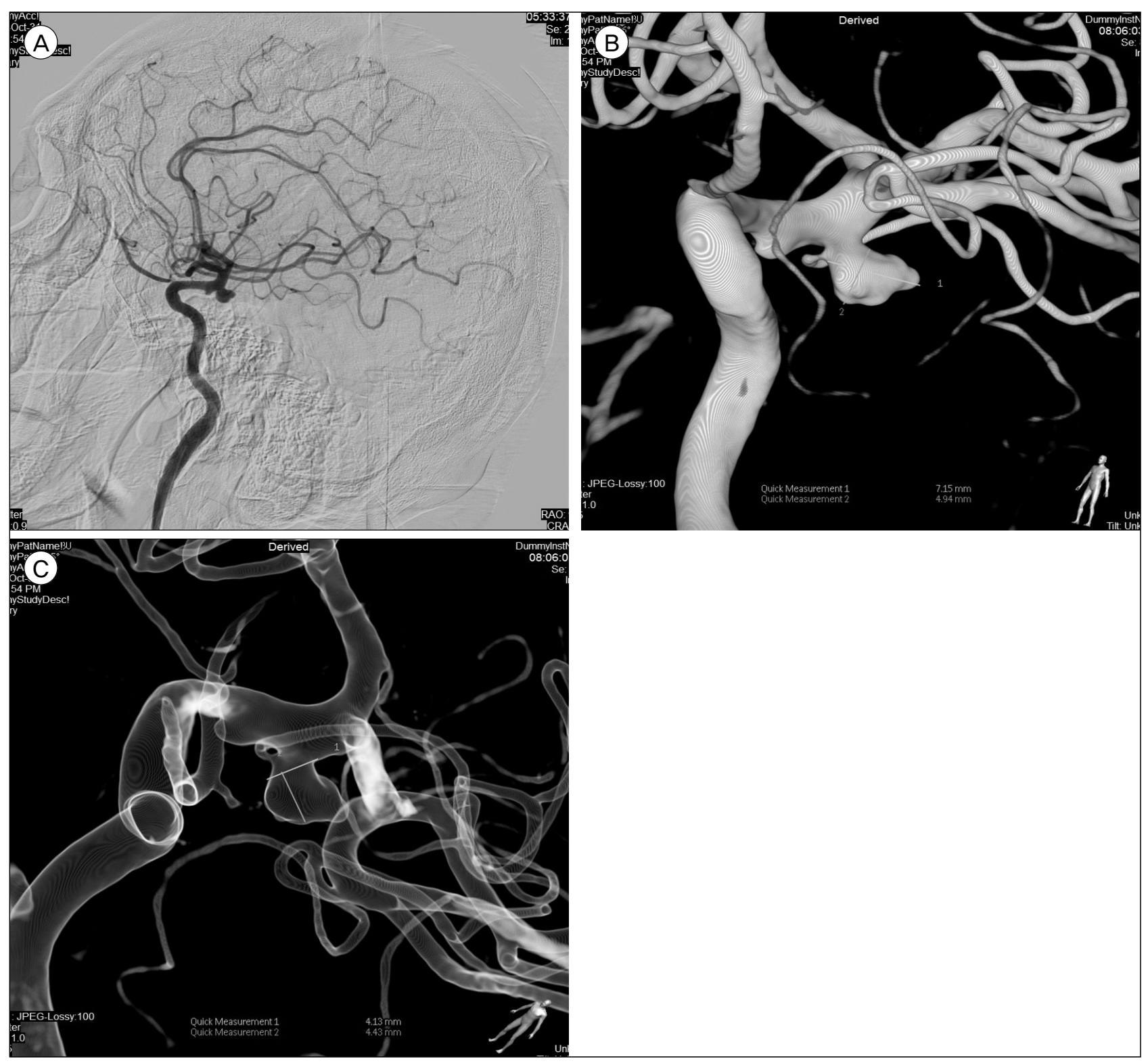

Fig. 3. Conventional cerebral angiogram clearly showing the aneurysm arising from the left anterior choroidal artery. A: Digital subtraction angiogram in the lateral view showing the left internal carotid artery. B and C: Three-dimentional reconstruction images showing the aneurysm more precisely.

procedure.

If IVH causes secondary obstructive hydrocephalus, EVD is recommended to facilitate blood clot washout, and CSF diversion is suggested to control the intracranial pressure (ICP). ${ }^{1}$ Other studies reported that a sharp increase in blood pressure and sharp decrease in ICP can induce rebleeding. ${ }^{17,22}$ Voldby and Enevoldsen proposed avoidance of CSF drainage until steady-state ICP was achieved to prevent recurrent hemorrhage. $^{22)}$ Therefore, if there is no significant neurological damage, CSF drainage should be considered after resolving the risk of rebleeding. Additionally, endovascular treatment can be performed sequentially after diagnostic angiography. Thus, the endovascular procedure is worthwhile treatment option in case of solitary IVH caused by aneurysmal rupture because it 


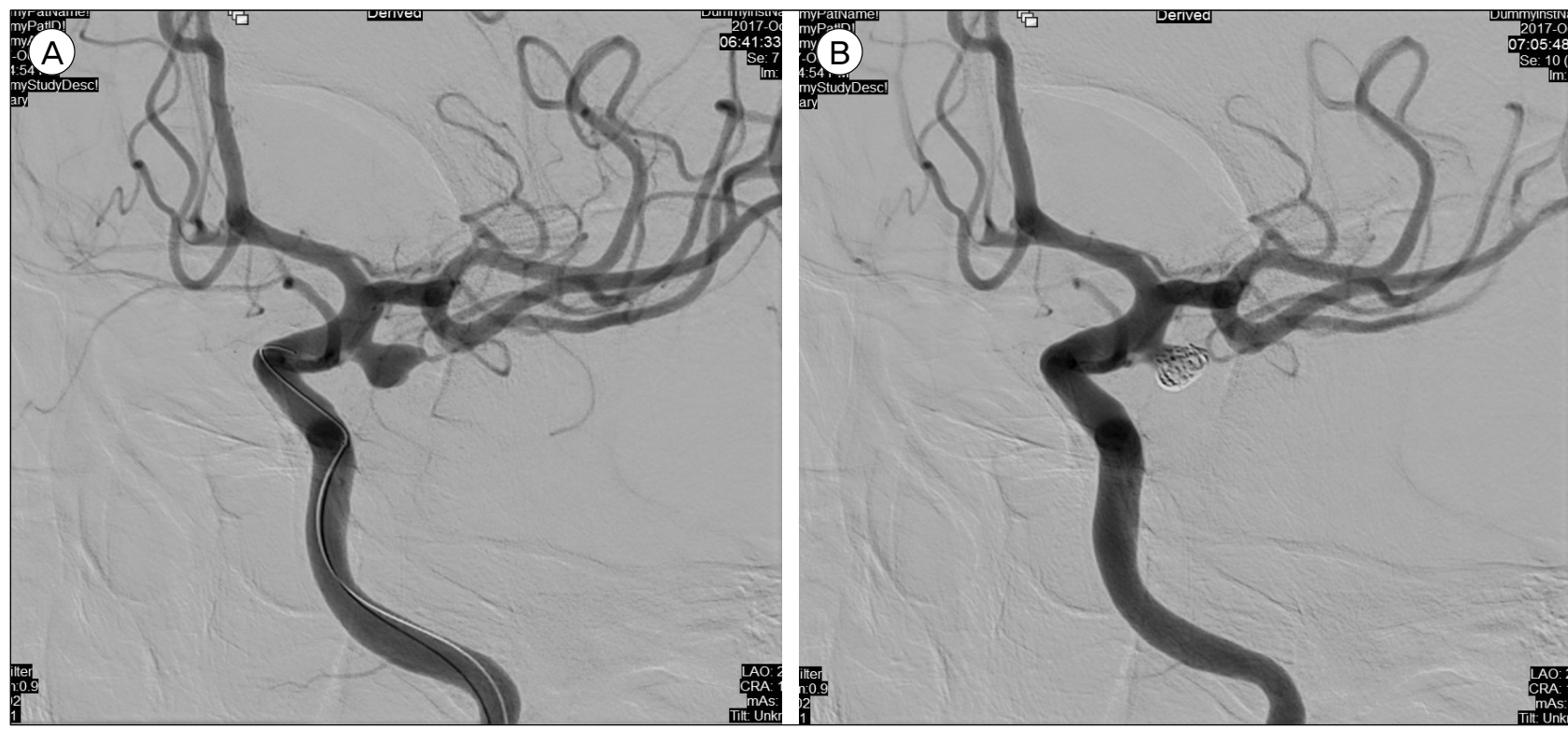

Fig. 4. A: Preoperative digital subtraction angiogram shows the endovascular catheter tip being advanced at the aneurysmal neck. B: Postoperative digital subtraction angiogram shows successful endovascular embolization and no emboli in distal blood flows.

requires a simple, sequential process after angiographic diagnosis compared to open surgery, and it can save time for the serial CSF diversion procedure. In our case, we performed coil embolization for ruptured cerebral aneurysm, which had a time-saving benefit and seemed to contribute to the patient's good prognosis.

\section{CONCLUSIONS}

In cases of primary IVH, CTA seems worthwhile for making a differential diagnosis, although the possibility of IVH due to cerebral aneurysmal rupture is very low. Endovascular treatment is another good option for primary IVH without SAH due to ruptured cerebral aneurysm.

\section{Funding}

We did not receive any funding.

Disclosure of Interest

The authors report no conflict of interest.

\section{REFERENCES}

1. Anagnostopoulos C., Chamilos C., Hadjigeorgiou G., Petsanas A. Isolated massive intraventricular haemorrhage without subarachnoid haemorrhage due to rupture of a posterior communicating artery aneurysm. Br J Neurosurg. 2017 Apr; 1-2.

2. Angelopoulos M., Gupta S.R., Kia B.A.. Primary intraventricular hemorrhage in adults: clinical features, risk factors, and outcome. Surg Neurol. 1995 May; 44(5), 433-436; discussion 437.

3. Darby D., Donnan G., Saling M., Walsh K., Bladin P. Primary intraventricular hemorrhage: clinical and neuropsychological findings in a prospective stroke series. Neurology. 1988 Jan; 38(1), 68-75.

4. Flint A.C., Roebken A. and Singh, V. Primary intraventricular hemorrhage: yield of diagnostic angiography and clinical outcome. Neurocrit Care. 2008 Mar; 8(3), 330-336.

5. Gates P.C., Barnett H. J.M., Vinters H.V., Simonsen R.L., Siu K. Primary intraventricular hemorrhage in adults. Stroke, 1986 Feb; 17(5), 872-877.

6. Inagawa T., Matsuda Y., Kamiya K., Aoyama H., Nagasako R., Yamamoto M. Saccular aneurysm of the distal anterior choroidal artery--case report. Neurol Med Chir (Tokyo). 1990 Mar; 30(7), 498-502.

7. Jayakumar, P. N., Taly A.B., Bhavani U.R., Arya B.Y.T., Nagaraja D. Prognosis in solitary intraventricular haemorrhage. Clinical and computed tomographic observations. Acta Neurol Scand 1989 Jul; 80(1), 1-5.

8. Knuckey N.W., Epstein M.H., Haas R., Sparadeo, F. Distal anterior choroidal artery aneurysm: intraoperative localization and treatment. Neurosurgery. 1988 Jun; $22(6$ 
Pt 1), 1084-1087.

9. Konishi Y., Kadowaki C., Hara M., Takeuchi K. Aneurysms associated with moyamoya disease. Neurosurgery. 1985;16(4), 484-491.

10. Martí-Fàbregas J., Piles S., Guardia E., Martí-Vilalta J. L. Spontaneous primary intraventricular hemorrhage: clinical data, etiology and outcome. J Neurol. 1999 Apr; 246(4), 287-291.

11. Nakagawa K., Lee S. B. Predominant intraventricular hemorrhage from a ruptured posterior communicating artery aneurysm. Neurohospitalist. 2014 Oct; 4(3), 164.

12. Nishihara J., Kumon Y., Matsuo Y., Sakaki S. A case of distal anterior choroidal artery aneurysm: case report and review of the literature. Neurosurgery. 1993 May; 32(5), 834-837; discussion 837.

13. Papo I., Salvolini U., Caruselli G. Aneurysm of the anterior choroidal artery with intraventricular hematoma and hydrocephalus. Case report. J Neurosurg. 1973 Aug; 39(2), 255-260.

14. Ragland R.L., Gelber N.D., Wilkinson H.A., Knorr, J.R., Tran A.A. Anterior communicating artery aneurysm rupture: an unusual cause of acute subdural hemorrhage. Surg Neurol. 1993; 40(5), 400-402.

15. Ranjan A., Joseph T. Giant aneurysm of anterior ethmoidal artery presenting with intracranial hemorrhage. Case report. J Neurosurg. 1994 Dec; 81(6), 934-936.

16. Roos Y. B., Hasan D., Vermeulen M. Outcome in patients with large intraventricular haemorrhages: a volumetric study. J Neurol Neurosurg Psychiatry. 1995 May; $58(5), 622-624$.

17. Rosenorn J., Westergaard L., Hansen P. H. Mannitol-induced rebleeding from intracranial aneurysm. Case report. J Neurosurg. 1983 Sep; 59(3), 529-530.

18. Sandin J.A., Salamat M.S., Baskaya M., Dempsey R.J. Intracerebral hemorrhage caused by the rupture of a nontraumatic middle meningeal artery aneurysm. Case report and review of the literature. J Neurosurg. 1999 May; 90(5), 951-954.

19. Tanaka Y., Ogashiwa M., Takeuchi K. Moyamoya phenomenon with accompanying intracranial aneurysm. Neuroradiology 1978 Dec; 16(1), 289-290.

20. Tembl J., Lago A., Baquero M., Blasco, R. Primary intraventricular hemorrhage: an analysis of eight cases. Rev Neurol. 1997; 25(138), 215-218.

21. Venti, M. Subarachnoid and intraventricular hemorrhage. Front Neurol Neurosci. 2012; 30, 149-153.

22. Voldby B., Enevoldsen E.M. Intracranial pressure changes following aneurysm rupture. Part 3: Recurrent hemorrhage. J Neurosurg. 1982 Jun; 56(6), 784-789.

23. Weinstein R., Ess K., Sirdar B., Song S., Cutting $S$. Primary Intraventricular Hemorrhage: Clinical Characteristics and Outcomes. J Stroke Cerebrovasc Dis. 2017 May; 26(5), 995-999.

24. Weisberg L.A. Computerized tomography in intracranial hemorrhage. Arch Neurol. 1979 Jul; 36(7), 422-426.

25. Yeung R., Ahmad T., Aviv R. I., de Tilly L. N., Fox A. J., Symons S. P. Comparison of CTA to DSA in determining the etiology of spontaneous ICH. Can J Neurol Sci. 2009 Mar; 36(2), 176-180.

26. Yoneoka Y., Ezuka I., Takai N., Oda T., Tamura T., Yamashita S. Ruptured distal anterior choroidal artery aneurysm presenting with casting intraventricular haemorrhage. Acta Neurochir (Wien). 1998 Feb; 140(2), 185-189. 